\title{
Potent Non-Hydroxamate Inhibitors of Histone Deacetylase-8: Role and Scope of an Isoindolin-2-yl Linker with an $\alpha$-Amino Amide as the Zinc-Binding Unit
}

\author{
Simon O. R. Greenwood, ${ }^{\mathrm{a}, \mathrm{b}}$ A. W. Edith Chan, ${ }^{\mathrm{c}}$ D. Flemming Hansen, ${ }^{\mathrm{b}}$ \\ and Charles M. Marson, *a
}

${ }^{a}$ Department of Chemistry, University College London, Christopher Ingold Laboratories, 20 Gordon Street, London WC1H OAJ, U.K. ${ }^{b}$ Division of Biosciences, Institute of Structural and Molecular Biology, University College London, London WCIE 6BT, U.K.

${ }^{c}$ Wolfson Institute of Biomedical Research, University College London, Gower Street, London WC1E 6BT, U.K.

\begin{abstract}
A series of potent inhibitors of histone deacetylase-8 (HDAC8) is described that contains an $\alpha$-amino amide zinc-binding unit and a substituted isoindolinyl capping group. The presence of a 2,4-dichlorophenyl unit located in the acetate-release cavity was shown to confer a gain of approx. $4.3 \mathrm{~kJ} \mathrm{~mol}^{-1}$ in binding energy compared to a phenyl group, and the isoindoline linker has approx. $5.8 \mathrm{~kJ} \mathrm{~mol}^{-1}$ greater binding energy than the corresponding tetrahydroisoquinoline ring system. In a series of 5-substituted isoindolin-2-yl inhibitors, a 5acetylamino derivative was found to be more potent than the 5-unsubstituted lead HDAC8 inhibitor (increase in binding energy of $2.0 \mathrm{~kJ} \mathrm{~mol}^{-1}$ ), ascribed to additional binding interactions within the $N_{\varepsilon}$-acetyl-L-lysine binding tunnel in HDAC8, including hydrogen bonding to Asp101. Tolerance of a 5-substituent (capping group) on the isoindoline ring has been demonstrated, and which in some cases confers improved enzyme inhibition, the HDAC8 substrate-binding region providing a platform for additional interactions.
\end{abstract}

Keywords: synthesis of isoindoline derivatives; $\alpha$-amido amide inhibitors; histone deactylase-8; ligand binding energies

Once considered as a post-translational modification unique to histones, lysine acetylation is now accepted as a common modification across the proteome. ${ }^{1}$ Histone deacetylases (HDACs) catalyse deacetylation of lysine residues, resulting in chromatin 
compaction and transcriptional repression ${ }^{2-4}$ where the substrates are histones. Increased levels of HDAC activity have been associated with precancerous or malignant states. Epigenetic mechanisms ${ }^{5}$ are increasingly implicated in cancer, heart disease and neurological disorders, and some inhibitors of histone deacetylase (HDAC) are used in cancer therapy, ${ }^{6,7}$ their relief of transcriptional repression ${ }^{8,9}$ playing an important role in the treatment of certain leukaemias. Of the Class I HDACs, HDAC1, HDAC2 and HDAC3 have been the main focus as targets for cancer treatment; however, upregulation of HDAC8 strongly correlates with the progression and prognosis of juvenile neuroblastoma ${ }^{10}$ which accounts for $15 \%$ of all pediatric deaths. ${ }^{11,12}$ HDAC 8 also plays a crucial role in the induction of the tumour suppressor p53, mutations of which are present in over $50 \%$ of all cancers. ${ }^{13}$ In a variety of cancer cell lines only knockdown of HDAC8, not knockdown of HDAC1, HDAC2 or HDAC3, led to decreased mutant and wild-type p53 expression; furthermore, disruption of HDAC8 function triggered defects in proliferation for cells with mutant but not wild-type p53. ${ }^{14}$ Thus, targeting HDAC8 selectively could provide a personalised therapy specifically for patients with cancers involving mutant p53. Consequently, structurally novel, specific inhibitors of HDAC8 are a priority.

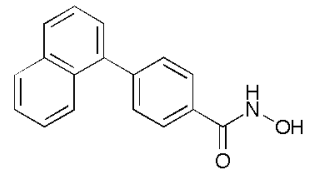

$1^{15}$

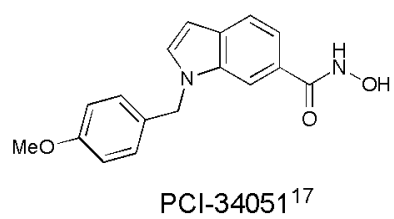

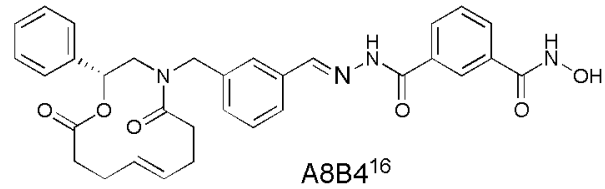

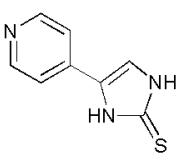

SB-379278A ${ }^{18}$

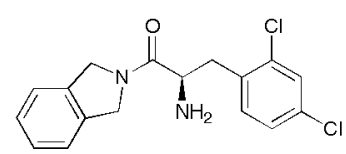

Novartis $\mathbf{2}^{19}$

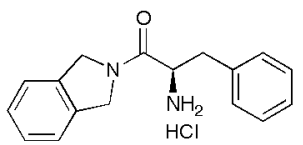

Figure 1. Examples of HDAC8-selective inhibitors.

Most Class I HDAC inhibitors used as anti-cancer agents ${ }^{6,7}$ are either hydroxamic acids such as $\mathbf{1}$, and include Vorinostat (suberoylanilide hydroxamic acid) ${ }^{20}$ or are aminoanilides such as Mocetinosat. ${ }^{21,22}$ The nonselective metal-chelating properties of hydroxamic acids confer limitations of efficacy and toxicity which restrict the scope of current HDAC 
inhibitors. Additionally, such inhibitors bind significantly in only three major regions: by coordination to zinc, by occupancy of the catalytic tunnel (that otherwise contains the lysine chain of the natural substrate), and through binding to the protein periphery (the Zn binderlinker-capping group assembly). Consequently, the report by a Novartis group of the $\alpha$ amino amide HDAC8 inhibitor 2 (Fig. 1) ${ }^{19}$ that binds in the acetate-release channel (located deeper within the protein, beyond the zinc-binding region) is an unusual feature that characterises this class of HDAC inhibitor, and has important implications for research programs on the discovery novel HDAC inhibitors, including our own research program that also aims to understand their mode of action. ${ }^{23,24}$ Herein are described the synthesis and preliminary evaluation of a new series of $\alpha$-amino amide HDAC8 inhibitors and a rationale for the structure-activity relationships observed.

HDAC8 inhibitor 2 is one of only two $\alpha$-amino amide HDAC inhibitors previously described, ${ }^{19}$ and to the best of our knowledge SAR studies have not been reported. To establish the SAR and gain greater understanding of such $\alpha$-amino amide ligands, the first series of compounds designed for synthesis and evaluation in the present work retained the isoindolin-2-yl linker, thereby permitting the energies of binding of various units in the acetate-release channel to be determined. Other initial aims were to establish whether replacements for the 2,4-(dichlorophenyl)methyl unit of $\mathbf{2}$ could be identified that increased the potency, and also to understand the unit's role and relative importance. In later series of $\alpha$-amino amides, we show here that whereas the isoindoline unit comprises part of the linker region, that a polar 5-substituent on that ring system can act as a capping group, engaging in hydrogen bonding with Asp101, as suggested by molecular docking.

To explore the scope of inhibitor structure binding in the acetyl-lysine region, analogs of 2 were prepared. In this work, the hydrochloride salts were synthesised and evaluated, and compared to inhibitor $\mathbf{5 q}$ (Scheme 1), the hydrochloride salt of $\mathbf{2}$. Coupling of carboxylic acid 3 with a variety of amines (aliphatic, benzylic, aromatic) all proceeded satisfactorily, giving the amides 4; Boc deprotection then gave the corresponding $\alpha$-amino amides 5 (Scheme 1). 


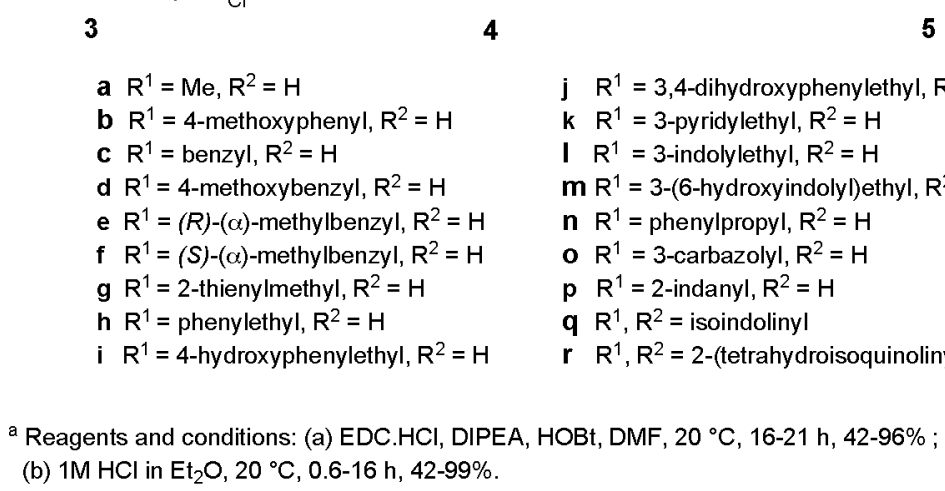

3

a $\mathrm{R}^{1}=\mathrm{Me}, \mathrm{R}^{2}=\mathrm{H}$

b $\mathrm{R}^{1}=4$-methoxyphenyl, $\mathrm{R}^{2}=\mathrm{H}$

c $\mathrm{R}^{1}=$ benzyl, $\mathrm{R}^{2}=\mathrm{H}$

d $\mathrm{R}^{1}=$ 4-methoxybenzyl, $\mathrm{R}^{2}=\mathrm{H}$

e $\mathrm{R}^{1}=(R)-(\alpha)$-methylbenzyl, $\mathrm{R}^{2}=\mathrm{H}$

f $\mathrm{R}^{1}=(S)-(\alpha)$-methylbenzyl, $\mathrm{R}^{2}=\mathrm{H}$

g $R^{1}=2$-thienylmethyl, $R^{2}=H$

h $\mathrm{R}^{1}=$ phenylethyl, $\mathrm{R}^{2}=\mathrm{H}$

i $\mathrm{R}^{1}=$ 4-hydroxyphenylethyl, $\mathrm{R}^{2}=\mathrm{H}$

4

a Reagents and conditions: (a) EDC. HCl, DIPEA, HOBt, DMF, $20^{\circ} \mathrm{C}, 16-21 \mathrm{~h}, 42-96 \%$;

(b) $1 \mathrm{M} \mathrm{HCl}$ in $\mathrm{Et}_{2} \mathrm{O}, 20^{\circ} \mathrm{C}, 0.6-16 \mathrm{~h}, 42-99 \%$.

Using the above route, a set of phenyl and 4-hydroxyphenyl derivatives was prepared (Scheme 2), mainly to determine the efficacy of the 2,4-dichloro groups in 5q. Compounds $\mathbf{8 c}$ and $8 \mathbf{e}$ are direct analogs of $\mathbf{5 q}$, having respectively no substituent and a 4-hydroxy group on the phenyl ring. However, in vitro assays of compounds 8a-8f showed lower inhibition of HDAC8 compared to $\mathbf{5 q}$, confirming the importance of the 2,4-dichlorophenylmethyl unit. One of the analogs $\mathbf{5 l}$ had an $\mathrm{IC}_{50}$ close to $1 \mu \mathrm{M}$ for inhibition of HDAC8, but none had improved potency compared to 5q. Overall, compounds containing an isoindoline ring in the series $\mathbf{8}$ were more potent than others that did not, and phenyl derivatives were more potent than 4-hydroxyphenyl derivatives.

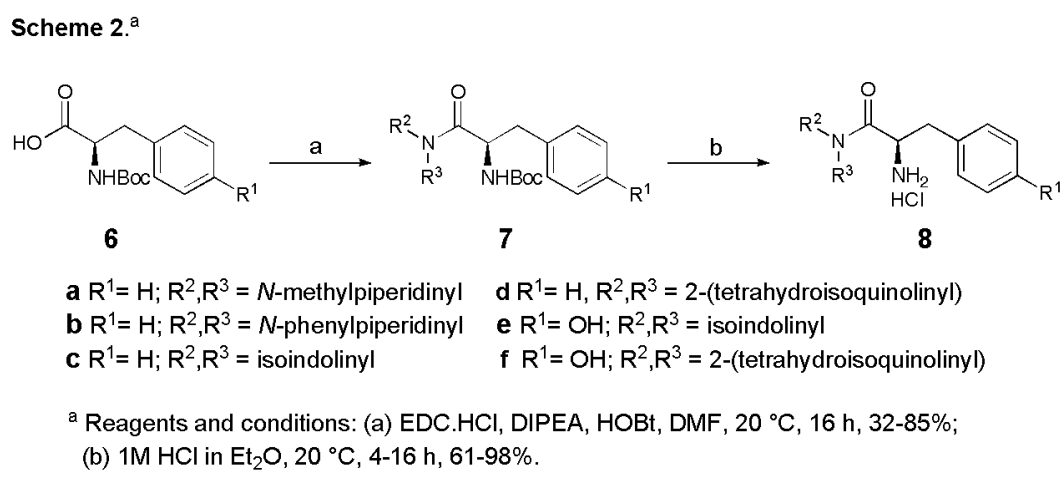

With indications of 2,4-dichlorophenylmethyl unit being a preferred structural motif, the second series of compounds focused on the introduction of a 5-substituent on the isoindoline ring (Schemes 3 and 4). (Ligand docking suggested that a 4-substituent would not be tolerated). Reduction of $\mathbf{9}^{25}$ (Scheme 3) furnished amine $\mathbf{1 0}$ from which three 5(acylamino)isoindolines were synthesised by a sequence of acylation of the 5-amino group, hydrogenolytic $N$-debenzylation, acylation at the 2-position, and Boc deprotection of the primary amino group to give the $\alpha$-amino amides 14b-14d (Scheme 3). The parent amine 
14a was synthesised by hydrogenolysis of 10 to give $(\mathbf{1 2 a})^{25}(51 \%)$ which underwent acylation with 3 to give 13a (44\%); subsequent deprotection gave $\mathbf{1 4 a}$.

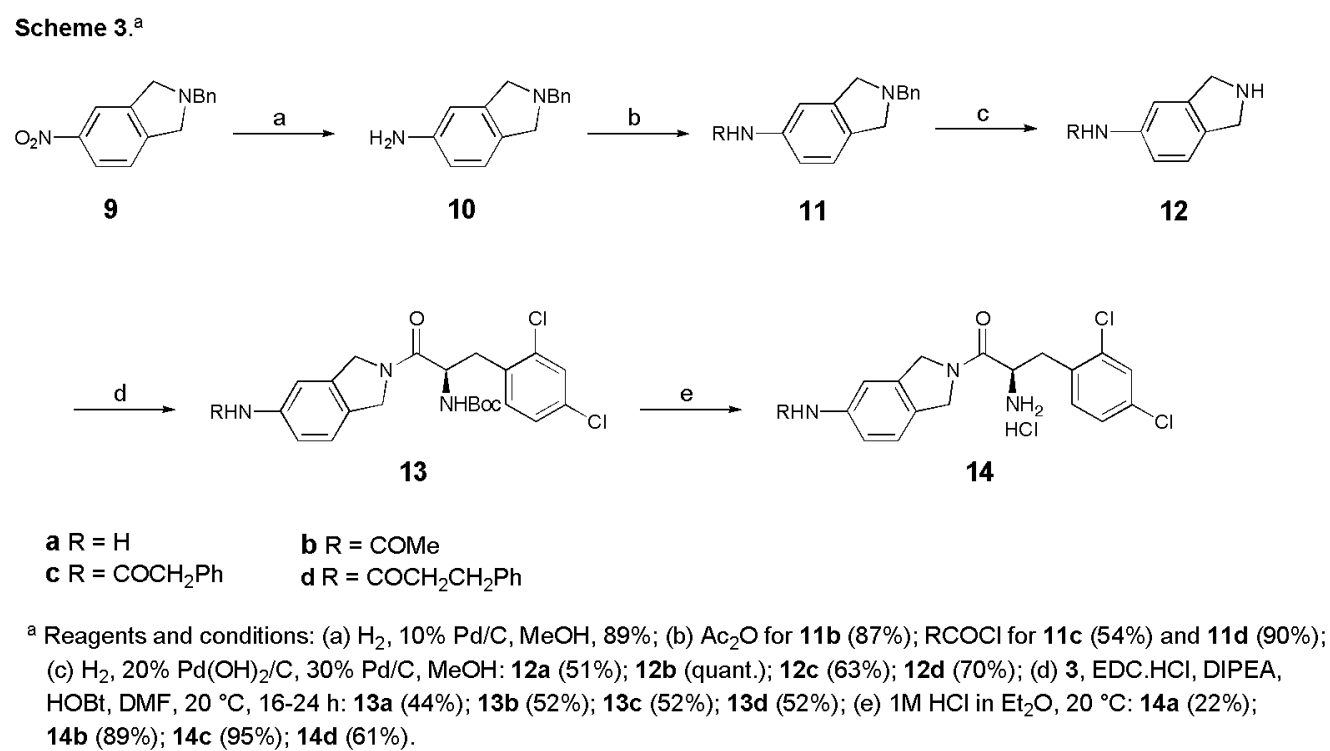

In order to maximise opportunities for hydrogen bonding of the 5-substituent on the isoindoline ring with HDAC8 residues, a route to the reverse amides was also devised and the representative $N$-methyl carboxamide 21 synthesised (Scheme 4). Reaction of bromoisoindoline-1,3-dione ${ }^{26,27}$ with benzylamine in acetic acid (1 h reflux) afforded 2benzyl-5-bromoisoindoline-1,3-dione ${ }^{27}$ (90\%) which underwent reduction with $\mathrm{NaBH}_{4}$ in the presence of $\mathrm{Et}_{2} \mathrm{O}_{3} \mathrm{BF}_{3}$ to give $1682 \%$ ). This reagent was found here to be generally satisfactory for the reduction of phthalimide derivatives to isoindoline derivatives; isoindoline itself has been prepared by the reduction of phthalimide with $\mathrm{THF} \cdot \mathrm{BH}_{3} \cdot{ }^{28}$ Metalation followed by carboxylation afforded the acid $\mathbf{1 7}$ which was converted into amide 18 and hence the $\alpha$-amino diamide 21.

Scheme $4 .^{a}$

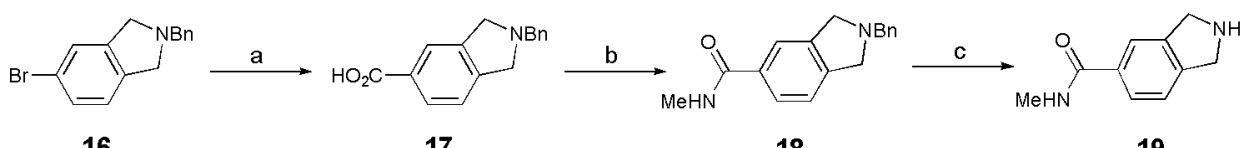

17

18

19

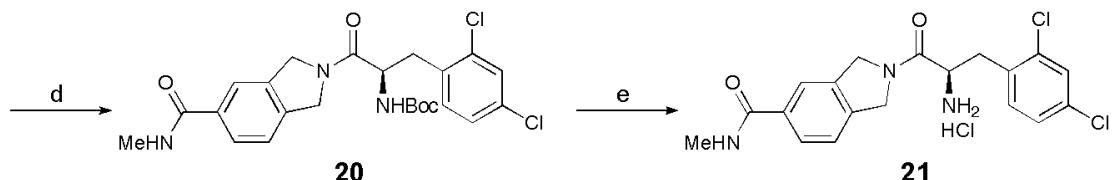

20

21

a Reagents and conditions: (a) $n$-BuLi, THF $\mathrm{CO}_{2}, 79 \%$; (b) $\mathrm{MeNH}_{2}, \mathrm{EDC} . \mathrm{HCl}, \mathrm{HOBt}, \mathrm{DMF}, 20^{\circ} \mathrm{C}, 74 \%$; (c) $\mathrm{H}_{2}, 20 \% \mathrm{Pd}(\mathrm{OH})_{2} / \mathrm{C}, 30 \% \mathrm{Pd} / \mathrm{C}, \mathrm{MeOH}, 28 \%$ with $45 \%$ of 18 recovered; (d) EDC, DIPEA, HOBt, DMF, $20^{\circ} \mathrm{C}, 36 \%$; (e) $1 \mathrm{M} \mathrm{HCl}$ in $\mathrm{Et}_{2} \mathrm{O}, 20^{\circ} \mathrm{C}, 95 \%$. 
Synthesis of 26, the amine analog of 21, required careful handling of disparate nitrogen functionality (Scheme 5). Reduction of amide $\mathbf{1 8}$ followed by $N$-Boc protection and debenzylation afforded the key protected amine 24. Acylation at the 2-position followed by concomitant deprotection of both Boc groups afforded the desired amine salt $\mathbf{2 6}$.

Scheme $5 .^{a}$

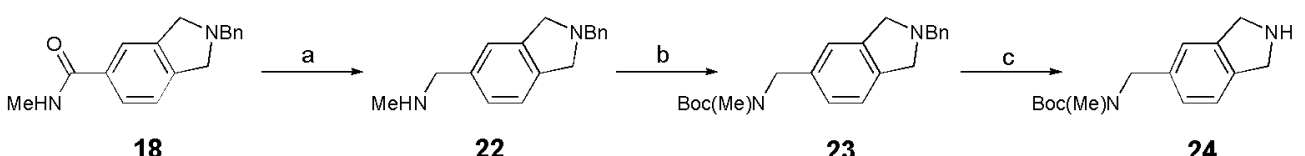

18

22

23

24

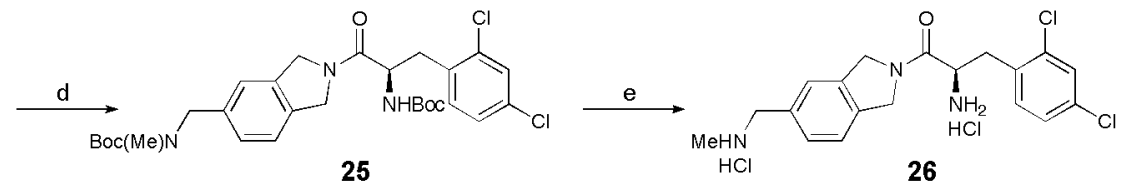

25

26

a Reagents and conditions: (a) $\mathrm{LiAlH}_{4}, \mathrm{THF}, \mathrm{O}^{\circ} \mathrm{C}$, then $23^{\circ} \mathrm{C}$, then reflux, $75 \%$; (b) (Boc) ${ }_{2} \mathrm{O}, t-\mathrm{BuOH}, 58 \%$;

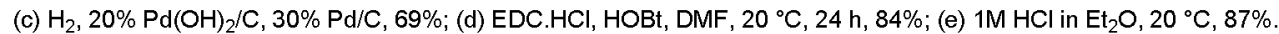

In order to probe HDAC8 inhibition, structural analysis and ligand binding, the initial set of compounds (Table 1) was designed to probe the binding energy of the isoindoline ring and the 2,4-dichlorophenyl unit, compared to other similar units. Since a diverse array of tetrahydroisoquinoline derivatives are straightforward to prepare, and are usually less prone to decomposition than isoindoline derivatives, the tetrahydroisoquinoline compounds $\mathbf{5 r}, \mathbf{8 d}$ and $\mathbf{8 f}$ were synthesised and evaluated for in vitro inhibition of HDAC8. The homolog of $\mathbf{5 q}$, compound $\mathbf{5 r}$, was tenfold less potent, showing that ring size and planarity are crucial to activity, probably in part because the larger ring departs from the planarity of the isoindoline ring which is evidently well accommodated in the acetyl-lysine binding tunnel. The efficacy of the 2,4-dichloro unit is shown in $\mathbf{5 r}$ being fivefold more potent than $\mathbf{8 d}$ (unsubstituted phenyl group) which in turn is twice as potent as $\mathbf{8 f}$ that contains a 4-hydroxy substituent. The same pattern was observed for the isoindoline series, $\mathbf{5 q}$ being fivefold more potent than 8c which in turn is more than three times as potent as 8e that contains a 4-hydroxy substituent. The fused aromatic ring of the tetrahydroisoquinoline series was evidently an indispensable feature since the $N$-methylpiperazine analog $8 \mathbf{a}$ was less potent than $\mathbf{5 r}$, and the $N$-methylpiperazine analog $\mathbf{8 b}$ showed no measurable activity. 


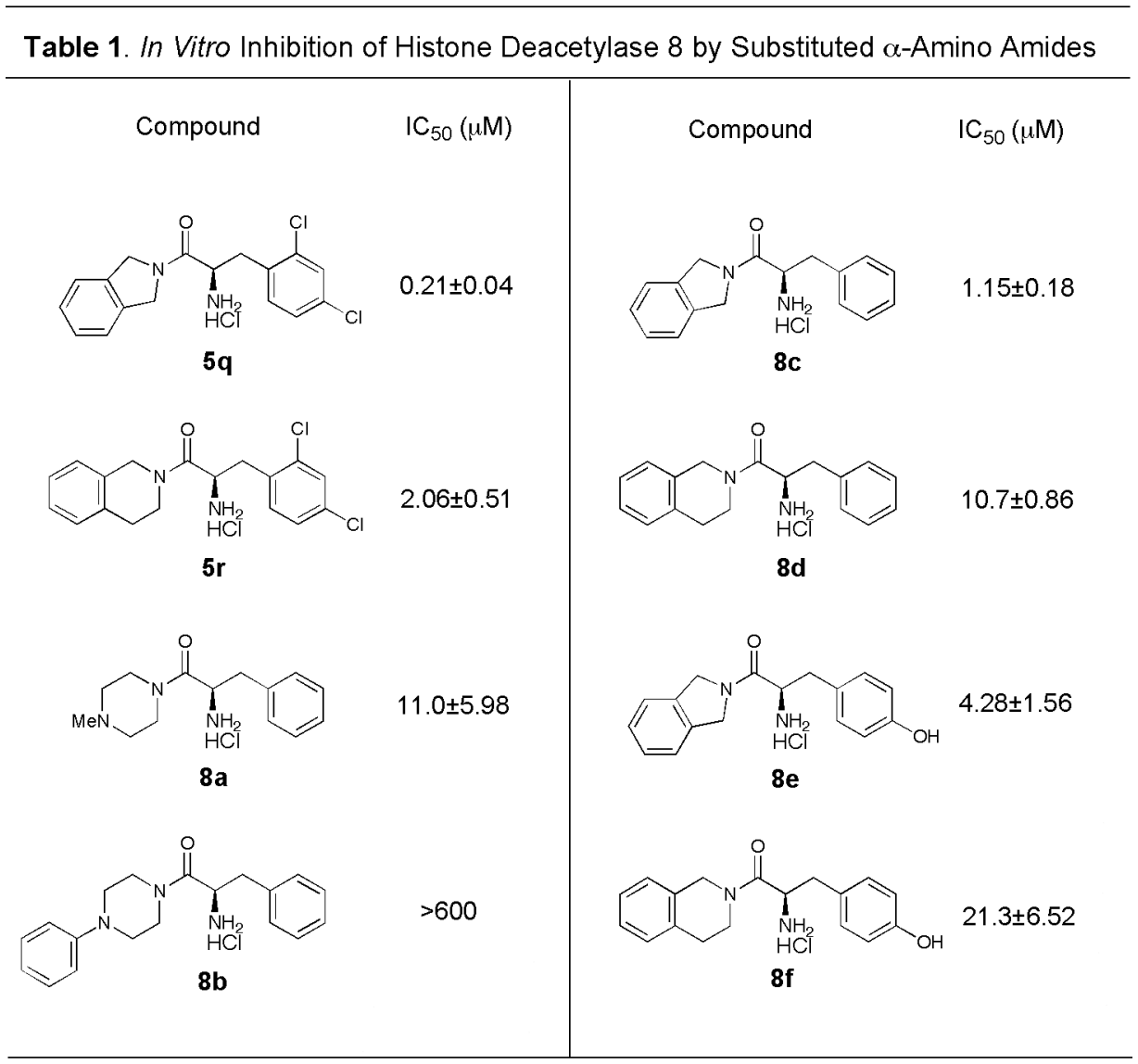

Since the results from the first series (Table 1) had not identified an improvement upon the 2,4-dichlorophenyl unit for binding, that unit was retained in the second series of compounds (Table 2) which probed interactions within the acetyl-lysine binding tunnel, a region associated with conformational flexibility, especially the ability of Phe152 to adopt a variety of orientations in order to accommodate different ligands. ${ }^{19}$ The Phe152 sidechain can rotate to reveal a pocket thought to be unique to HDAC8. ${ }^{19}$ The parent amino acid (Table 2, first entry) and the methyl carboxamide 5a, compounds lacking a region that could bind in the acetyl-lysine binding tunnel, were some of the least potent inhibitors of HDAC8. Chain extension, either as an aryl ring (5b) or as a benzyl substituent (5c) restored potency to low micromolar levels. The loss of $7.5 \pm 1.7 \mathrm{~kJ} \mathrm{~mol}^{-1}$ binding energy (see Supplementary Material) on going from the conformationally constrained ring in $\mathbf{2}$ to the unrestricted benzyl group in 5c corresponds to the expected increase in entropy of $2.5-4.2 \mathrm{~kJ} \mathrm{~mol}^{-1}$ per rotatable bond gained, ${ }^{29}$ and suggests that any additional poses of the non-fused benzene ring offer no advantage in binding. An $\alpha$-methylbenzyl group attached to the terminal nitrogen atom was not well tolerated, although the $(R)$-enantiomer $5 \mathbf{d}$ was threefold more potent than the $(S)$ enantiomer 5e. A $p$-methoxy group had an adverse effect upon HDAC inhibition (compare 5f 
to $\mathbf{5 c}$ ). A 2-thienyl group (5g) showed a small increase in potency compared to the benzyl analog (5c). The phenylethyl series $\mathbf{5 h}-\mathbf{5 j}$ exhibited low micromolar potency, the 4-hydroxy group being well tolerated, and possibly conferring some potency. A 3-phenylpropyl unit (5n) also showed low micromolar potency, but a 2-indanyl $N$-substituent (5p, an analog of $\mathbf{5 h}$ constrained to form a ring), was less beneficial than any of the linear analogs. The presence of nitrogen within the $\mathrm{N}$-substituent was advantageous in the case of indolylethyl analogs (5l and $\mathbf{5 m}$ ), the parent compounds showing potency close to $1 \mu \mathrm{M}$. However, a 3-carbazolyl substituent conferred lower potency than an aromatic analog (compare $\mathbf{5 p}$ to $\mathbf{5 b}$ ), probably because of steric bulk. Overall, while some benzylic and $N$-substituents were well tolerated, no improvement was identified as superior to the 2-isoindolin-2-yl unit. 
Table 2. In Vitro Inhibition of Histone Deacetylase 8 by Varied-Linker $\alpha$-Amino Amides

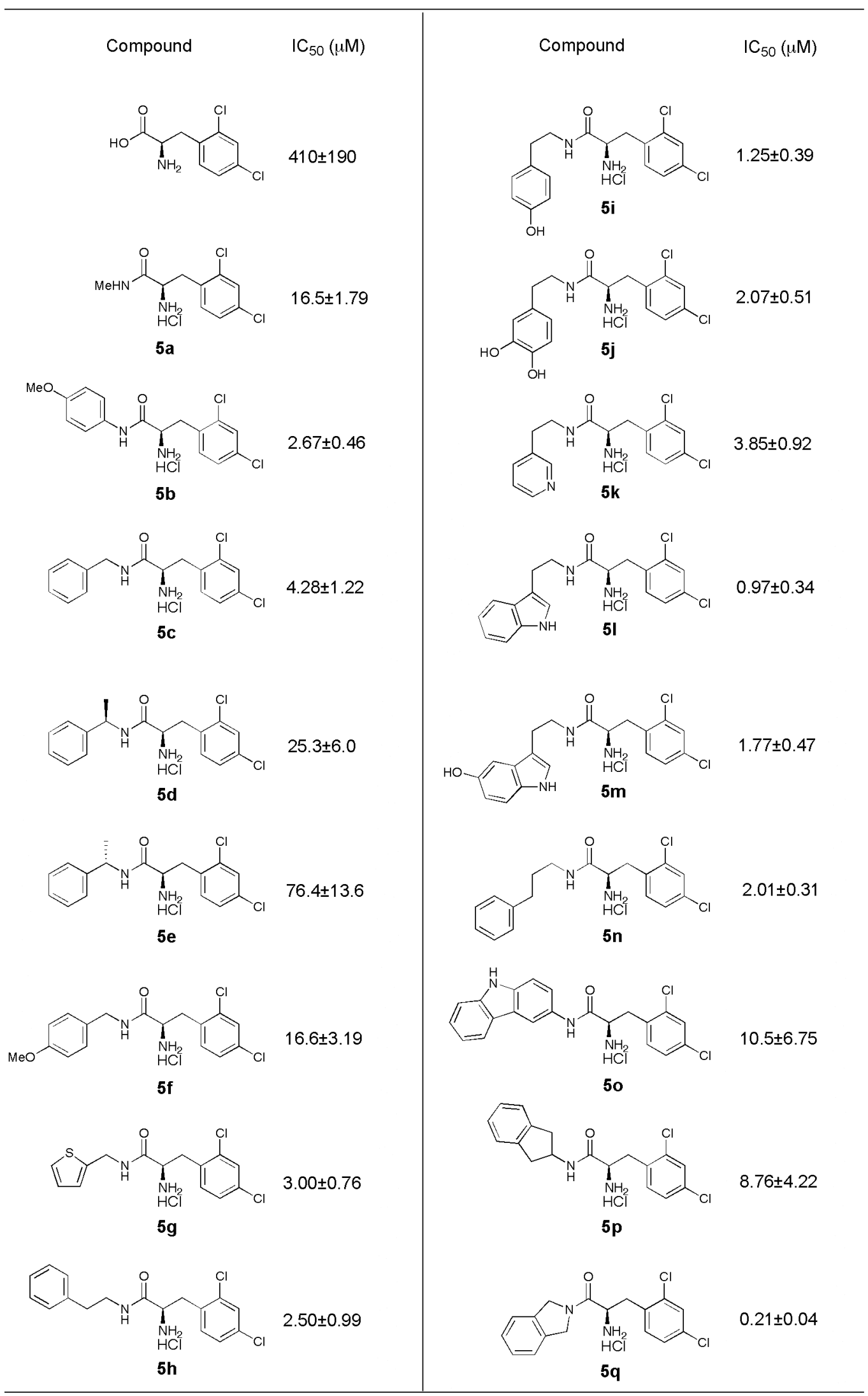

Rigidity and a planar ring system linked linearly (w.r.t. the N-CO bond) appear to be the main factors that confer greater potency of isoindolin-2-yl derivatives compared to other 
systems. Compared to $\mathbf{5 q}$, the greater number of rotatable bonds in $\mathbf{5 c}, \mathbf{5 h}$ and $\mathbf{5 n}$ conferred much lower binding to HDAC8 (Table 2).

Inhibitory values of HDAC8 for previous compounds, especially those in Table 2, confirmed a strong preference for an isoindolin-2-yl unit to be present in the linker unit. Accordingly, this ring system was studied in the final series of compounds synthesised (Table 3). Lateral substitution (4- or 4,7-positions) was not considered since preference for a relatively linear and planar ring system in the catalytic tunnel was strongly indicated by molecular docking (discussed below) and also supported by the above in vitro HDAC8 inhibition assay data. Since the synthesis of substituted isoindolines can pose considerable challenges, efforts were focused on preparing 5-acylamino and 5-aminoacyl derivatives, potentially conferring hydrogen-bond donor or acceptor properties, or even both, to provide additional interactions with the enzyme. Encouragingly, HDAC8 inhibition compared to $\mathbf{5 q}$ was greater for the parent 5-aminoisoindoline 14a and for the corresponding $N$-acetyl derivative 14b, and was comparable or better for all other compounds synthesised in this series (Table 3). The amides containing phenylethanoyl and 3-phenylpropanoyl $\mathrm{N}$ substituents (14c and $\mathbf{1 4 d}$ respectively), of comparable potency to $\mathbf{5 q}$, show potential for further optimisation through the incorporation of substituents on the aryl rings. Reversal of the amide linkage in 14b still retained the high level of potency, although HDAC8 inhibition of amide $\mathbf{2 1}$ was about one-half that of $\mathbf{1 4 b}$. The $N$-methylaminomethyl derivative $\mathbf{2 6}$ showed comparable or improved potency compared to amide $\mathbf{2 1}$ and all other compounds in the series. 
Table 3. In Vitro Inhibition of HDAC8 by Substituted Isoindolinyl $\alpha$-Amino Amides

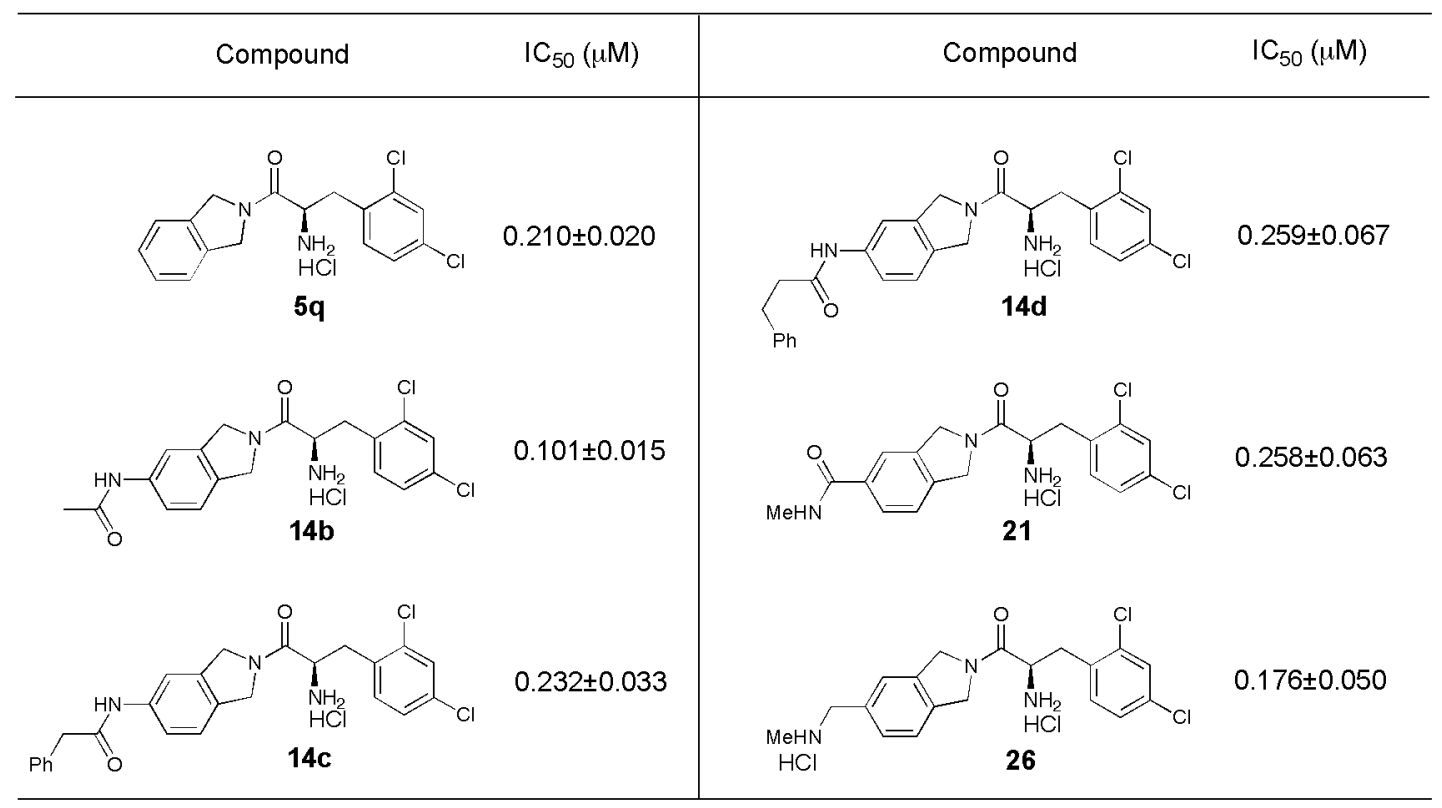

Interpretation of the above results in terms of both HDAC8-ligand crystallography and molecular docking is insightful. For all the $\alpha$-amino amides herein discussed, including 2 (apparently of the same constitution as 5q), binding in the acetate-release channel (located deeper in the protein, beyond the zinc-binding region; Fig. 2) is an unusual feature that characterises this class of HDAC inhibitor. Among the 11 human HDAC isoforms, Trp at position 141 is unique to HDAC8, and being present within the acetate-release channel, is thought to play a major role in the selectivity of inhibitor binding to HDAC $8,{ }^{30}$ involving aromatic stacking, as confirmed by crystallography of amide 2 in HDAC8 (Fig. 2a). Offcentre parallel aromatic stacking occurs between the 2,4-dichlorophenyl unit of inhibitor 2 and Trp141. Interactions of the 2-chloro substituent with Gly140, and of the 4-chloro substituent with Arg37 also contribute to binding in the acetate-release channel (Fig. 2a). Arg37 has been shown to regulate the release of acetate. ${ }^{31,32}$ The 4-chloro substituent interacts side-on to Arg37, a standard mode for a Lewis base. In contrast, the 2-chloro substituent is end-on to the backbone carbonyl oxygen atom of Gly140, and is engaged in halogen bonding. ${ }^{33}$

For all the $\alpha$-amino amides in Table 3 , the similar or improved potency of the 5substituted isoindoline derivatives can be accounted for as follows. In many HDAC isoforms other than HDAC8 a capping group that binds to the protein periphery is required for potent inhibition. The potential for improved binding by adding a capping group to $\mathbf{2}$ is also supported by previous findings that Asp101 is essential for substrate binding in HDAC8 via the formation of a hydrogen bond. ${ }^{34,35}$ Such hydrogen bonding involving Asp101 is shown in 
Figs. $2 b$ and 2c, whereas in the crystal structure of 2 the flexible location of Asp101 is not resolved, suggesting that it does not bind to $\mathbf{2}$. Given that amides $\mathbf{5 i}$ and $\mathbf{5 1}$ (containing polar $\mathrm{OH}$ and $\mathrm{NH}$ groups respectively that could engage in hydrogen bonding) were the most potent in the series shown in Table $2\left(\mathrm{IC}_{50}\right.$ values close to $\left.1 \mu \mathrm{M}\right)$ in which the isoindoline ring is absent, this supported examining the effect of the introduction of a 5-substituent in an isoindoline series that might also engage in hydrogen bonding with Asp101.

The 5-substituted isoindoline series (Table 3) provided further evidence of effectiveness in exploiting ligand binding to Asp101; for the most potent compounds, amine 14a $\left(\mathrm{IC}_{50}=0.13 \mu \mathrm{M}\right)$ and amide $14 \mathbf{b}\left(\mathrm{IC}_{50}=0.10 \mu \mathrm{M}\right)$, molecular docking poses (Figs. $2 \mathrm{~b}$ and 2c) clearly identified distances consistent with hydrogen bonding to Asp101 (approximately $2.75 \AA$ in both cases) as well as an aromatic edge-to-face interaction with Phe152. The NH groups of the 5-NHR substituent act as hydrogen bond donors to the Asp101 sidechain (Fig. 2). However, translocation of the nitrogen atom to the $\beta$-position of the 5-substituent, as in the reversed amide $\mathbf{2 1}$ and the aminomethyl derivative $\mathbf{2 6}$ lowers the ligand binding energy. In amide 21, the amide is more than $4 \AA$ from Asp101 and the orientation of the $\mathrm{N}-\mathrm{H}$ bond departs from the ideal linear hydrogen-bonded assembly. ${ }^{36}$
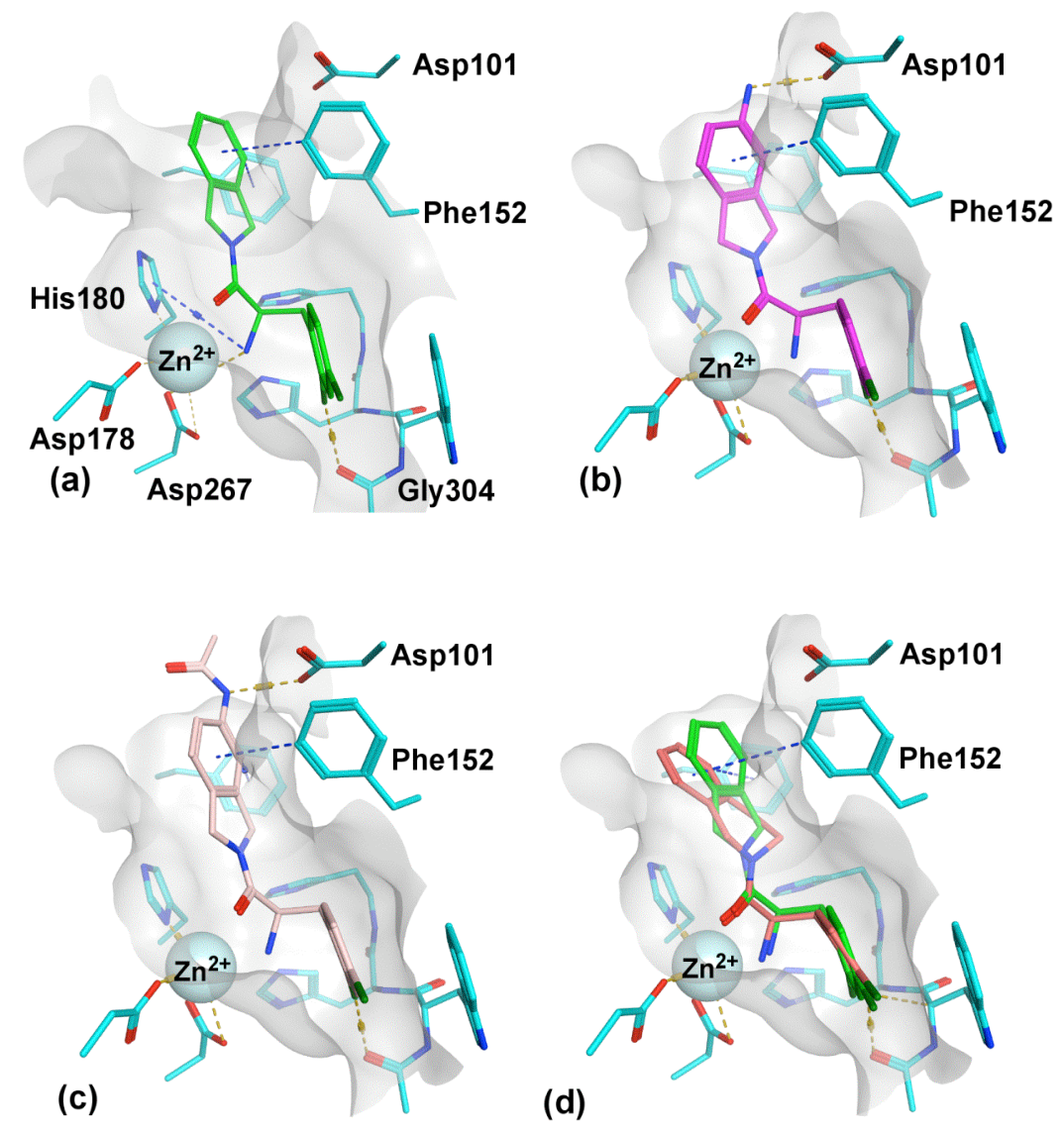
Figure 2. (a) Conformation of $\alpha$-amino amide 2 in HDAC8 (PDB: 3 SFH). ${ }^{19}$ (b)-(d) Poses of $\alpha$-amino amides 14a, 14b and 5r respectively docked into HDAC8 (PDB: 3SFH, see supplementary material). In addition to edge-to-face interactions of the aromatic ring of isoindoline, (b) and (c) also indicate hydrogen bonding to Asp101 of the amine substituent in 14b (in magenta), and the amide substituent 14c (in pink) respectively. Figure (d) shows the markedly different pose of $\mathbf{5 r}$ (brown) containing a fused piperidine ring compared to $\mathbf{2}$ (overlaid in green).

As shown in the crystal structure (PDB: $3 \mathrm{SFH}),{ }^{19}$ the aromatic ring of isoindoline in 2 is well positioned for edge-to-face interactions with Phe152 of HDAC8, but that is not the case for the corresponding tetrahydroisoquinolinyl analog $\mathbf{5 r}$, being more remote from Phe152 (Fig. 2d); additionally, the different topology of a larger and a non-planar isoquinoline ring would lead to altered interactions with backbone amide residues. Such factors would contribute to the lower binding energy compared to the isoindoline ring system in 2 , as reflected by a difference of $5.8 \mathrm{~kJ} \mathrm{~mol}^{-1}$ estimated from $\mathrm{IC}_{50}$ values. The lack of aromatic interactions and a relatively planar but non-aromatic ring also account for the weak binding of the piperazine derivatives $\mathbf{8 a}$ and $\mathbf{8 b}$.

In conclusion, general routes to 5-substituted isoindolines have been developed, and their conversion into the corresponding $\alpha$-amino amides has furnished potent HDAC8 inhibitors. Amide 14b was found to be highly selective for HDAC8 $\left(\mathrm{IC}_{50}=0.10 \mu \mathrm{M}\right)$, compared to HDAC1 $\left(\mathrm{IC}_{50}=8.8 \mu \mathrm{M}\right)$, HDAC2 $\left(\mathrm{IC}_{50}>30 \mu \mathrm{M}\right)$, and HDAC6 $\left(\mathrm{IC}_{50}>30\right.$ $\mu \mathrm{M})$. The presence of the 2,4-dichloro substitution at the phenyl ring of the $\alpha$-amino amides was shown to confer a gain of approx. $4.3 \mathrm{~kJ} \mathrm{~mol}^{-1}$ in binding energy, being an essential unit in this series of HDAC8 inhibitors, for binding within the acetate-release channel. ${ }^{19}$ The need for a linear, planar and rigid ring system, as indicated by the efficacy of a isoindolin-2-yl linker, was underscored by its replacement with tetrahydroisoquinoline ring system (approx. $5.8 \mathrm{~kJ} \mathrm{~mol}^{-1}$ lower binding energy than the isoindoline ring system) which invariably conferred poorer in vitro inhibition of HDAC8. A final series of $\alpha$-amino amides was synthesised and shown to give potent in vitro inhibition of HDAC8, compounds 14a and 14b being somewhat more potent than the lead HDAC8 inhibitor $\mathbf{5 q}$ in the assay used, with improved binding energy of about $2.0 \mathrm{kcal} \mathrm{mol}^{-1}$ arising from additional binding interactions within the acetyl-lysine binding tunnel, including $\pi-\pi$ interactions, particularly involving 
Phe152, and hydrogen bonding of a 5-isoindoline substituent to Asp101. The importance of Asp101 in HDAC8 activity and its inhibition has been previously emphasised; $;^{30,34,35}$ mutations of Asp101 led to loss of inhibitor binding in studies of hydroxamic acid inhibitors of HDAC $8 .^{30}$

This study has evaluated the scope and limitations of indolin-2-yl $\alpha$-amino amides as HDAC8 inhibitors, and has provided an analysis of binding energies of the various structural features along with rationales for the binding of each major structural subunit. Appropriately substituted isoindolinyl inhibitors have been shown to confer additional binding of the HDAC8 inhibitor to the acetyl-lysine binding tunnel, the substituent acting as a capping group that offers additional molecular diversity. Consequently, there is scope for the development of more potent and isoform-selective inhibitors of HDACs containing an $\alpha$-amino amide as the zinccoordinating unit within the active site.

*Corresponding author: E-mail: c.m.marson@ucl.ac.uk FAX:+44(0)20 76797463

\section{Acknowledgments}

Financial support from the BBSRC for a studentship (to S. O. R. G.) is gratefully acknowledged. D. F. H. acknowledges the BBSRC for financial support (BB/H022570/1). We are grateful to Dr Christopher Matthews for providing the MAL substrate and to Drs Nicolas D. Werbeck and Micha B. A. Kunze for assistance with in vitro assays and dockings, and for helpful discussions.

\section{Supplementary data}

Supplementary data for this article can be found at...

\section{Conflicts of interest}

There are no conflicts to declare.

\section{References}

(1) Kouzarides T. EMBO J. 2000; 19; 1176.

(2) Jenuwein T, Allis, CD. Science 2001; 293: 1074.

(3) Taunton J, Hassig, CA, Schreiber, SL. Science 1996; 272: 408. 
(4) Hassig CA, Schreiber SL. Curr. Opin. Chem. Biol. 1997; 1: 300.

(5) Strahl BD, Allis CD. Nature 2000; 403: 41.

(6) Marson CM. Anti-Cancer Agents in Medicinal Chemistry 2009; 9: 661.

(7) Minucci S, Pelicci G. Nat. Rev. Cancer 2006; 6: 38.

(8) Mahlknecht U, Hoelzer D. Mol. Med. 2000; 6: 623.

(9) Gayther SA, Batley SJ, Linger L, Bannister A, Thorpe K, Chin SF, Daigo Y, Russell P, Wilson A, Sowter HM, Delhanty JD, Ponder BA. J, Kouzarides T, Caldas C. Nat. Genet. 2000; 24: 300.

(10) Oehme I, Deubzer HE, Wegener D, Pickert D, Linke J-P, Hero B, Kopp-Schneider A, Westermann F, Ulrich SM, von Deimling A, Fischer M, Witt O. Clin. Cancer Res. 2009; 15: 91.

(11) Brodeur GM Nat. Rev. Cancer 2003; 3: 203.

(12) Maris JM, Hogarty MD, Bagatell R, Cohn SL, Lancet 2007; 369: 2106.

(13) Liu DP, Song H, Xu Y. Oncogene 2010; 29: 949.

(14) Yan W, Liu S; Xu E, Zhang J, Zhang Y, Chen X. Oncogene 2013; 32: 599.

(15) KrennHrubec K, Marshall BL, Hedglin M; Verdin E, Ulrich SM. Bioorg. Med. Chem. Lett. 2007; 17: 2874.

(16) Tang W, Luo T, Greenberg EF, Bradner JE, Schreiber SL. Bioorg. Med. Chem. Lett. 2011; 21: 2601.

(17) Balasubramanian S, Ramos J, Luo W, Sirisawad M, Verner E, Buggy JJ. Leukemia 2008; 22:1026.

(18) Hu E, Dul E, Sung C-M, Chen Z, Kirkpatrick R, Zhang G-F, Johanson K, Liu R, Lago A, Hofmann G, Macarron R, De Los Frailes M, Perez P, Krawiec J, Winkler J, Jaye M. J. Pharmacol. Exp. Ther. 2003; 307: 720.

(19) Whitehead L, Dobler MR, Radetich B, Zhu Y, Atadja PW, Claiborne T, Grob JE, McRiner A, Pancost MR, Patnaik A, Shao W, Shultz M, Tichkule R, Tommasi RA, Vash B, Wang P, Stams T. Bioorg. Med. Chem. 2011; 19: 4626.

(20) (a) Richon VM, Garcia-Vargas J, Hardwick JS. Cancer Lett. 2009; 280: 201. (b) Kelly WK, O’Connor OA, Krug ML, Chiao JH, Heaney M, Curley T, MacGregoreCortelli B, Tong W, Secrist JP, Schwartz L, Richardson S, Chu E, Olgac S, Marks PA, Scher H, Richon VM. J. Clin. Oncol. 2005; 23: 3923.

(21) Zhou N, Moradei O, Raeppel S, Leit S, Frechette S, Gaudette F, Paquin I, Bernstein N, Bouchain G, Vaisburg A, Jin Z, Gillespie J, Wang J, Fournel M, Yan PT, Trachy- 
Bourget M-C, Kalita A, Lu A, Rahil J, MacLeod AR, Li Z, Besterman JM, Delorme D. J. Med. Chem. 2008; 51: 4072.

(22) Garcia-Manero G, Minden M, Estrov Z, Verstovsek S, Newsome WM, Reid G, Besterman J, Li Z, Pearce L, Martell R. J. Clin. Oncol. 2006; 24: 6500.

(23) Marson CM, Matthews CJ, Yiannaki E, Atkinson SJ, Soden PE, Shukla L, Lamadema N, Thomas NSB. J. Med. Chem. 2013; 56: 6156.

(24) Marson CM, Matthews CJ, Atkinson SJ, Lamadema N, Thomas NSB. J. Med. Chem. 2015; 58: 6803.

(25) Carpino PA, Dasilva-Jardine PA, Lefker BA, Ragan JA. US Patent 5936089, 1999, August 10, Pfizer Inc.

(26) Chytil M, Engel SR, Fang QK, Spear KL. US Patent 204214, 2010, August 10.

(27) Blanck S, Maksimoska J, Baumeister J, Harms K, Marmorstein R, Meggers E. Angew. Chem. Int. Ed. 2012; 51: 5244.

(28) Anastasiadis C, Hogarth G, Wilton-Ely JDET. Inorganica Chim. Acta 2010; 363: 3222.

(29) Chang CA, Chen W, Gilson MK. Proc. Natl. Acad. Sci. 2007; 104: 1534.

(30) Weerasinghe SVW, Estiu G, Wiest O, Pflum MKH. J. Med. Chem. 2008; 51: 5542.

(31) Haider S, Joseph CG, Neidle S, Fierke CA, Fuchter MJ. Bioorg. Med. Chem. Lett. 2011; 21: 2129.

(32) Kalyaanamoorthy S, Chen Y-P. J. Comput. Chem. 2013; 34: 2270.

(33) Wilcken R, Zimmermann MO, Lange A, Joerger AC, Boeckler FM. J. Med. Chem. 2013; 56: 1363.

(34) Vannini A, Volpari C, Gallinari P, Jones P, Mattu M, Carfí A, De Francesco R, Steinkühler C, Di Marco S. EMBO Rep. 2007; 8: 879.

(35) Kunze MBA, Wright DW, Werbeck ND, Kirkpatrick J, Coveney PV, Hansen DF. J. Am. Chem. Soc. 2013; 135: 17862.

(36) Williams MA, Ladbury JE in Protein-Ligand Interactions: From Molecular Recognition to Drug Design. Eds. Böhm H-J, Schneider G. Wiley-VCH, 2003, pp. 137-161. 


\section{TOC Graphic}
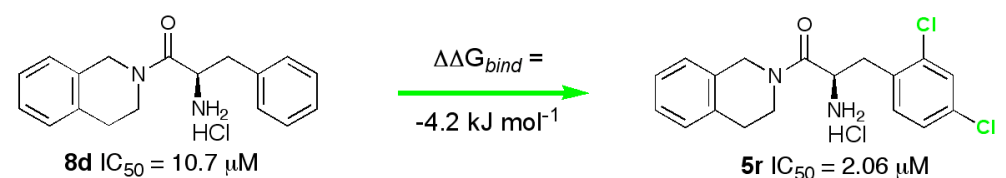

$5 \mathrm{r} I \mathrm{C}_{50}=2.06 \mu \mathrm{M}$
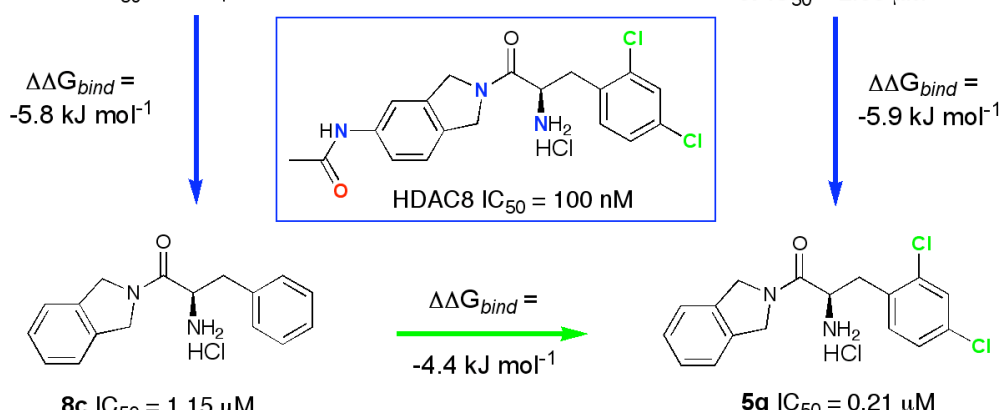

$8 \mathrm{c} I \mathrm{I}_{50}=1.15 \mu \mathrm{M}$

$5 q \mathrm{IC}_{50}=0.21 \mu \mathrm{M}$ 
